\title{
Intermediación de conocimiento: proceso de la gestión del conocimiento en cooperativas agropecuarias venezolanas
}

\author{
Flores Urbáez, Matilde* \\ Ramírez Omaña Rosa**
}

\section{Resumen}

En este artículo se analiza la intermediación del conocimiento (subproceso de la gestión de conocimiento) en cooperativas agropecuarias del municipio La Cañada de Urdaneta, estado Zulia, Venezuela. La investigación fue exploratoria-descriptiva aplicándose la estadística descriptiva. Los principales resultados revelan que en la intermediación de conocimiento, los métodos de almacenamiento de conocimiento que poseen las cooperativas son de acceso restringido (intermediación asincrónica) lo que obstaculiza la socialización del conocimiento entre sus integrantes. Sin embargo, aunque de manera moderada, están presentes elementos de intermediación sincrónica asociados al intercambio de conocimiento tácito, como lo son las reuniones de trabajo.

Palabras clave: Gestión del conocimiento, intermediación del conocimiento, cooperativas agropecuarias.

\section{Knowledge Intermediation: The Knowledge Management Process in Venezuelan Agricultural Cooperatives}

\author{
Abstract \\ This article analyzes the intermediation of knowledge (a subprocess of knowledge manage- \\ ment) in agricultural cooperatives in the Municipality La Cañada de Urdaneta, State of Zulia, Vene- \\ Recibido: 09-12-10. Aceptado: 12-07-11 \\ * Doctora en Ciencias Sociales. Profesora Asociada adscrita a la Unidad Académica Estudios \\ del Desarrollo del Departamento de Ciencias Humanas de la Universidad del Zulia. Venezuela. \\ E-mail: floresurbaez8@yahoo.com \\ ** MSc en Gerencia de Proyectos de Investigación y Desarrollo. Supervisora del Sistema de \\ Información Geográfica del Instituto Nacional de Tierras (INTI). Maracaibo, estado Zulia. \\ Venezuela. E-mail: rosacramirez@yahoo.es
}


zuela. The research was exploratory-descriptive, applying descriptive statistics. Principle results reveal that in knowledge intermediation, the cooperatives have restricted access (asynchronic intermediation) knowledge storage methods, which create obstacles for socializing knowledge among the members. However, elements of synchronic intermediation are present, although in a moderate manner, associated with the exchange of tacit knowledge, as in work meetings.

Key words: Knowledge management, knowledge intermediation, agricultural cooperatives.

\section{Introducción}

A partir de la entrada en Vigencia la Ley Especial de Asociaciones Cooperativas, el 18 de septiembre de 2001, en Venezuela se multiplicó la conformación de empresas cooperativas, principalmente motivadas por los incentivos fiscales (exención de impuestos), créditos flexibles y acceso a contratos preferenciales.

Este importante apoyo tiene su origen en la Constitución de la República Bolivariana de Venezuela (1999), la cual en los artículos 70, 118, 184 y 308 reconoce el derecho de los trabajadores a organizarse en este tipo de estructura, obliga a formular leyes y políticas públicas que favorezcan su creación, funcionamiento y financiamiento, además de declarar la obligación que tiene el Estado de protegerlas y asegurar la capacitación de sus socios, la asistencia técnica y el financiamiento oportuno.

Sustentado en ese mandato constitucional, en 2001 se formula la Ley Especial de Asociaciones Cooperativas (LEAC) con el fin de establecer las regulaciones que se derivan de la Constitución. Tiene carácter de Ley Especial y su objeto es establecer las normas generales para la organización y funcionamiento de las cooperativas, disponer los mecanismos de relación, participación e integración de dichos entes en los procesos comunitarios con los sectores públicos, privados y con la economía social y participativa, constituida por las empresas de carácter asociativo que se gestionan en forma democrática.

A partir de este impulso legal y financiero, el auge en la creación de cooperativas es significativamente notorio, pues según la Superintendencia Nacional de Cooperativas (Sunacoop), de 820 cooperativas en 1999, pasó a 181.000 cooperativas a mediados de 2007.

la LEAC, que las conceptualiza como asociaciones abiertas y flexibles, de hecho y derecho cooperativo, de la economía social y participativa, autónomas, de personas que se unen mediante un proceso y acuerdo voluntario, para hacer frente a sus necesidades y aspiraciones económicas, sociales y culturales comunes, para generar bienestar integral, colectivo y personal, por medio de procesos y empresas de propiedad colectiva, gestionadas y controladas democráticamente.

A pesar del pleno apoyo que el Estado venezolano le otorga a las cooperativas, a través del mandato constitucional de protección, promoción, capacitación, asistencia técnica y financiamiento a las cooperativas con miras a impulsar la economía social como fuente generadora de empleo y de bienestar; la LEAC establece en sus artículos 82 y 85 aspectos relacionados con la Superintendencia Nacional de Cooperativas (Sunacoop) como ente controlador y fiscalizador. 
Intermediación de conocimiento: proceso de la gestión del conocimiento ...

Flores Urbáez, Matilde y Ramírez Omaña Rosa

Por otra parte, el actual escenario económico y social venezolano y mundial representa un reto para las cooperativas. Su razón social debe ser sustentable, lo que implica pasearse por las distintas leyes, elementos del mercado (oferta, demanda, equilibrio de mercado), la formación de sus socios, el respeto por el ambiente, la responsabilidad social, entre otros, para ofrecer bienes y servicios a consumidores que exigen de alta calidad y bajos costos.

Para incentivar la producción venezolana, para 2005 el Fondo de Crédito Industrial (FONCREI), actualmente Instituto Nacional de Desarrollo de la Pequeña y Mediana Industria, otorgó 257 millones 667 mil bolívares fuertes en créditos a cooperativas socio productivas de todo el país, con un total 235 créditos. Para 2006 se otorgaron 369 créditos por una suma de 154 millones 485 mil bolívares fuertes, para un total de 756 empleos directos. En 2007 el Fondo otorgó más de 80 mil bolívares fuertes en créditos al sector productivo venezolano, de los cuales fueron beneficiarias más de 400 cooperativas. Lo anterior representa una fuerte suma de dinero en apoyo las políticas de Estado al incentivo de la producción nacional y el desarrollo endógeno (MINCI, 17-03-2008).

Por otra parte, la Comisión de Administración de Divisas (Cadivi), del 1ero de enero al 31 de diciembre de 2007, autorizó un total de 5.843 millones de dólares para la adquisición de alimentos (MINCl, 4-1-2011), lo cual representó una interesante oportunidad de negocio para las cooperativas agropecuarias. De las 181.000 cooperativas registradas en Venezuela para el año 2007 según cifras de Sunacoop, 274 corresponden a coopera- tivas de ahorro con 125 mil asociados y manejan recursos por el orden de los ochenta millardos de bolívares (Sunacoop, 26-09-2007).

Estas cifras confirman un fuerte apoyo económico gubernamental que permite a las cooperativas comenzar con cierta ventaja financiera respecto a la empresa privada, por considerarlas protagonistas del crecimiento de la economía social del país.

Dada la relevancia de la seguridad alimentaria en las actuales políticas del gobierno venezolano (véase Plan Nacional de Ciencia, Tecnología e Innovación 2005-2030 y Proyecto Nacional Simón Bolívar 2007-2013), la fuerte inyección de recursos financieros, y el marco jurídico que respalda a las cooperativas, es muy probable que se haya incrementado la cantidad de cooperativas en este ramo.

Estas cifras sirven de referencia para afirmar que estas organizaciones tienen un fuerte apoyo económico del Estado, que en teoría les permite comenzar con cierta ventaja financiera respecto a las empresas privadas. Tomando en cuenta la importancia que para el gobierno de Venezuela tiene el sector cooperativista dentro del crecimiento de la economía social del país y dada la relevancia de la seguridad alimentaria en las actuales políticas del gobierno venezolano, queda clara la importancia de estudiar la gestión del conocimiento en cooperativas agropecuarias, como herramienta organizacional que puede servir de base para implementar mejoras en los procesos, hacerlos sustentables y agregar valor a sus productos y servicios.

En este trabajo se analizó el subproceso de intermediación de conoci- 
miento, el cual es útil para coordinar factores intangibles que facilitan, entre otras cosas, la actualización permanente de sus asociados y la revisión de los procesos cognitivos que marcan el comportamiento de los individuos, basados en sus creencias personales.

Este análisis se hará desde las teorías de la gestión del conocimiento, partiendo del modelo teórico de Koulopoulos y Frappaolo (2000), específicamente.

Esta investigación puede definirse como exploratoria porque, aun cuando existen estudios empíricos relacionados con la gestión del conocimiento en cooperativas, siguen siendo escasos. Por otra parte, el estudio fue de tipo descriptivo debido a que se recolectaron datos sobre dimensiones o componentes que permitieron abordar en detalle el fenómeno a investigar. Se definieron las unidades y subunidades temáticas y se recolectó información sobre cada una de ellas, para así proceder a describir el fenómeno investigado (Hernández y otros, 2006).

Se aplicó como técnica de campo la encuesta y como instrumento una entrevista dialógica de 21 ítems. Las categorías de respuesta fueron preestablecidas para que los informantes clave pudieran seleccionar tanto una, como varias o todas, dependiendo de las características de la organización. Para el análisis de los datos recolectados se calculó la frecuencia relativa y absoluta de cada una de las respuestas obtenidas de los informantes clave.

El diseño de la investigación fue no experimental, de tipo transeccional o transversal porque se recolectaron datos en un tiempo único, con el propósito de analizar la gestión del conocimiento en cooperativas agropecuarias del Munici- pio La Cañada de Urdaneta en un momento dado.

La población estuvo integrada por nueve (9) unidades de análisis, representadas por cooperativas agropecuarias, activas, en plena producción, ubicadas en el municipio La Cañada de Urdaneta del estado Zulia. Los informantes clave de cada cooperativa, 15 en total, fueron socios dispuestos a participar en el estudio, tomando en consideración su apertura hacia el suministro de información y disponibilidad de tiempo.

Debido a la posibilidad de tener acceso a toda la población, no se realizó muestreo sino que se trabajó con la población completa. Esto es lo que Méndez (2002) y Hernández y otros (2006) denominan censo.La validez del instrumento utilizado para la recolección de información en campo, fue de contenido y se realizó a través del juicio de 5 expertos.

Para complementar la validez de contenido realizada, se les suministraron los instrumentos a dos socios de una cooperativa agropecuaria del municipio Jesús Enrique Losada que no formaban parte de la población de investigación, para verificar que los enunciados y las instrucciones se comprendían (prueba piloto). Con base a los resultados de la validación de los expertos y de las observaciones hechas por los asociados a las cooperativas, se reformuló y posteriormente se aplicó el instrumento.

\section{El cooperativismo: algunas consideraciones teóricas}

Aunque existen vestigios de relaciones cooperativas en épocas más remotas, esa forma de asociatividad tomó 
Intermediación de conocimiento: proceso de la gestión del conocimiento ...

Flores Urbáez, Matilde y Ramírez Omaña Rosa

fuerza durante la revolución industrial. El movimiento cooperativo se originó como una forma de organización alternativa de los trabajadores, que buscaban solventar su situación de escasos ingresos, adoptando para ello una serie de esquemas y principios de cooperación (Zabala, 2007; en Bustamante, 2009).

La Rochdale Equitable Pioneers Society es la institución universalmente reconocida como pionera del cooperativismo (Monzón, 2003; en Bustamante, 2009). Esta sociedad fue constituida en el año de 1844, por veintiocho tejedores de las fábricas de Rochdale (Inglaterra) que habían quedado sin trabajo tras una huelga y que unieron esfuerzos para abrir un almacén, estableciendo para su administración unas reglas conocidas como los Principios de Rochdale o Principios Cooperativos, los cuales aún tienen vigencia, pues fueron acogidos desde 1937 por la Alianza Cooperativa Internacional -ACI(Bustamante, 2009).

La $\mathrm{ACl}$ es una organización no gubernamental fundada en Londres en el año de 1895, que agrupa a la mayoría de organizaciones cooperativas en todo el mundo, con el propósito de crear entre ellas una red económica internacional y promover el cooperativismo. La misma, participa en la Organización de las Naciones Unidas (ONU) en calidad de órgano consultivo. En el año de 1995, en la celebración del XXXI Congreso de la ACI llevado a cabo en Manchester (Reino Unido), los principios cooperativos fueron reformulados, dando origen a la Declaración sobre Identidad Cooperativa en la cual se establecieron los Principios Cooperativos del Siglo XXI, en los que se definen las cooperativas y se enuncian los valores y principios sobre los que se sustentan estas organizaciones.

De acuerdo con la declaración mencionada, una cooperativa es "una asociación autónoma de personas que se han unido de forma voluntaria para satisfacer sus necesidades y aspiraciones económicas, sociales y culturales en común, mediante una empresa de propiedad conjunta y gestión democrática" (ACl, 1995).

Para cumplir con este propósito, las cooperativas se basan en los valores de la autoayuda, la autoresponsabilidad, la democracia, la igualdad, la equidad y la solidaridad. De igual modo, los principios que deben seguirse para poner en práctica dichos valores son: la adhesión voluntaria y abierta; la gestión democrática por parte de los asociados; la participación económica de los asociados; la autonomía e independencia; la educación, formación e información; la cooperación entre cooperativas y el interés por la comunidad (ACl, 1995).

Una cooperativa de trabajo asociado (CTA) es una organización sin ánimo de lucro, regida por los principios cooperativos, que vincula el trabajo personal de sus asociados y sus aportes para satisfacer sus necesidades mediante la producción de bienes o la prestación de servicios y les permite participar de manera democrática en la gestión.

Según la Declaración Mundial sobre Cooperativismo de Trabajo Asociado, aprobada en 2005, por la Asamblea General del Comité Ejecutivo de la Organización Internacional de Cooperativas de Producción Industrial Artesanal y de Servicios (CICOPA), este tipo de organizaciones debe tener las siguientes características básicas: 
- Tener como propósito crear y mantener puestos de trabajo sustentables, que generen riqueza, mejoren la calidad de vida y promuevan el desarrollo comunitario y local; se basan en la adhesión libre y voluntaria de los socios.

- El trabajo debe estar a cargo de los socios.

- La relación entre el asociado y la cooperativa se rigen por regímenes concertados democráticamente, y son autónomas ante el Estado y terceros en lo referente al trabajo, la gestión y la disposición y manejo de los medios de producción.

De acuerdo con las características enunciadas, las cooperativas de trabajo asociado son una forma particular del modelo cooperativo, con una naturaleza jurídica y organizativa especial, que las diferencia de otras organizaciones y que tiene repercusiones en el modo de definir y concebir las estrategias.

Al igual que en otros ámbitos de la actividad económica, las cooperativas constituyen el eje básico de la economía social tanto por su importancia empresarial como por las características propias de su organización, que las configuran como empresas con funcionamiento $y$ gestión democráticos y supeditación a lo social.

Particularmente, las cooperativas agrarias son estructuras asociativas, cuya génesis está ligada al desarrollo de actividades en el ámbito de la producción, el comercio, las finanzas y las prestaciones de servicios; todo ello con el fin de mejorar las condiciones de vida de los productores agropecuarios en el medio rural. Nacen para defender los intereses de los peque- ños y medianos productores, ante las dificultades que ha padecido el sector y que se manifiestan recientemente en una baja obtención de ingresos (rentas), un constante aumento de los precios de los factores de producción, y una disminución de las ayudas institucionales o subvenciones (Fransi et al., 2007).

En estos tiempos de complejas transformaciones, la fórmula cooperativa mantiene plena vigencia debido a que una cooperativa estrechamente ligada a su entorno social y geográfico adquiere un papel importante en el desarrollo económico de los pueblos y ciudades. Es necesario que en la búsqueda de soluciones concretas a los problemas que tienen planteados las cooperativas, se respeten los principios cooperativos, puesto que la pérdida de sus señas de identidad harían más vulnerables a dichas entidades a la hora de afrontar su futuro.

El escenario estratégico sobre el cual deberán actuar las cooperativas agrarias en un futuro inmediato, está vinculado a modificaciones de la Política Agrícola Común (PAC) y cambios en el marco de las negociaciones en la Organización Mundial del Comercio (OMC), lo que previsiblemente generará mayores desequilibrios territoriales al liberalizar y aumentar la competitividad en los mercados agrarios, favorable a la presencia de transnacionales agroalimentarias que actúan como oligopolios en el sector productivo, comercial y de distribución. En la práctica, fomentar y dar apoyo a las cooperativas agrarias significa apostar por un desarrollo sostenido del medio rural en su vertiente económica, social y medioambiental (Fransi et al., 2007). 
Intermediación de conocimiento: proceso de la gestión del conocimiento ...

Flores Urbáez, Matilde y Ramírez Omaña Rosa

La complejidad de la situación socioeconómica actual plantea la necesidad de que dichas entidades asuman nuevos objetivos, tales como la mejora de la calidad y la conservación del medio ambiente, nuevas estrategias de ventas, diversificación de la producción, gestión del conocimiento, entre otros. En este último aspecto nos enfocaremos en el presente artículo.

\section{Enfoques de la gestión del conocimiento}

La gerencia del conocimiento es un catalizador del aprendizaje colectivo y de la innovación, además de ser relevante para identificar las condiciones del entorno que estimulen la acción individual y organizacional en respuesta a condiciones favorables o desfavorables.

Para autores como Martín Rubio y De La Calle (2003), la gestión del conocimiento es la función que planifica, coordina y controla los flujos de conocimiento que se producen en la empresa en relación con sus actividades y esenciales. Nonaka y Takeuchi (1995) consideran que la gestión del conocimiento es la ca- pacidad de la empresa para crear conocimiento nuevo, diseminarlo en la organización e incorporarlo en productos, servicios y sistemas.

Los conceptos anteriormente expuestos y sus distintos enfoques se resumen en el Cuadro 1.

La importancia de la gestión del conocimiento para las organizaciones radica en que ésta constituye una herramienta para generar valor a todos y cada uno de los procesos organizacionales. Distintos autores de la literatura especializada en temas gerenciales, así lo confirman.

Koulopoulos y Frappaolo (2000) consideran que la gestión del conocimiento es la herramienta fundamental para apoyar el crecimiento de las organizaciones en función de las condiciones cambiantes del entorno, donde lo importante es prepararse para responder a cualquier situación no planificada. Todo esto es posible tomando en cuenta que el punto de partida es la sabiduría colectiva, el conocimiento de cada uno como un todo, para lograr la innovación que es lo que finalmente determina la competitividad.

Cuadro 1

Cuadro comparativo: concepto de gestión del conocimiento

\begin{tabular}{|c|c|c|}
\hline Autor & Concepto & Enfoque \\
\hline $\begin{array}{l}\text { Koulopoulos y } \\
\text { Frappaolo } \\
(2000)\end{array}$ & $\begin{array}{l}\text { Apalancamiento de la sabiduría colectiva } \\
\text { para aumentar la capacidad de respuesta y la } \\
\text { innovación. }\end{array}$ & $\begin{array}{l}\text { Centrado en la sabiduría } \\
\text { colectiva. }\end{array}$ \\
\hline $\begin{array}{l}\text { Martín Rubio } \\
\text { y De La Calle } \\
\text { (2003) }\end{array}$ & $\begin{array}{l}\text { Planificar, coordinar y controlar los flujos de } \\
\text { conocimiento en relación con sus actividades } \\
\text { esenciales. }\end{array}$ & $\begin{array}{l}\text { En concordancia con las } \\
\text { actividades esenciales de } \\
\text { la organización. }\end{array}$ \\
\hline $\begin{array}{l}\text { Nonaka } \\
\text { y Takeuchi } \\
\text { (1995) }\end{array}$ & $\begin{array}{l}\text { Crear conocimiento nuevo, diseminarlo en la } \\
\text { organización e incorporarlo en productos, } \\
\text { servicios y sistemas. }\end{array}$ & $\begin{array}{l}\text { Aplicación del conocimien- } \\
\text { to existente para crear nue- } \\
\text { vo conocimiento. }\end{array}$ \\
\hline
\end{tabular}

Fuente: Elaboración propia. 
Desde el punto de vista de Martín Rubio y De La Calle (2003), la importancia de la gestión del conocimiento está en la generación y no en la acumulación de conocimiento. Por su parte, Cope (2001, en Sanguino 2003) apunta a tres procesos de gestión del conocimiento: el descubrimiento, la codificación y la difusión. En el descubrimiento del conocimiento se amplía la cantidad y calidad de nuestro almacén de conocimiento a cabo a través de procesos como la lectura, escritura, conferencias y trabajo en equipo.

El objetivo de la codificación es colocar al conocimiento en alguna forma entendible y organizada, para que pueda ser utilizado por todas las personas que necesiten de él. Los conocimientos explícitos son codificados con mayor facilidad. Sin embargo, por su complejidad y por residir en la mente de las personas, habiendo sido desarrollados y asimilados básicamente por experiencias, la codificación del conocimiento tácito no siempre es posible. Una forma de proveer un mayor acceso a este tipo de conocimiento es la elaboración de un mapa de conocimiento donde se encuentre, dentro de la organización, el conocimiento que se precisa. Este mapa apunta a las personas, documentos, bancos de datos, puede ser usado como índi- ce de conocimiento o como herramienta para evaluar el stock corporativo de conocimiento organizativo. La difusión del conocimiento consiste en compartir esquemas mediante un proceso de interacciones de colaboración y retos. Es el proceso de compartir conocimiento cuyo objetivo es ampliar o mejorar el valor y la calidad del contenido y no permutar y comercializar su valor en el mercado abierto (Cope, 2001; en Sanguino, 2003).

Para Nonaka y Takeuchi (1995) la importancia de la gestión del conocimiento se centra en su creación por parte de la empresa, que luego lo transmite a toda la organización de manera tal que se convierta en mejoras de los productos y servicios que ofrecen.

En el Cuadro 2 se resume la importancia de la gestión del conocimiento desde la perspectiva de los autores anteriormente señalados.

Para los fines de este artículo, se adopta la postura de Koulopoulos y Frappaolo (2000), tomando en cuenta su inclinación hacia la importancia de la sabiduría colectiva, la cual deja ver la generación de conocimiento en equipo, punto clave para el crecimiento de las organizaciones de naturaleza cooperativista. Por otra parte, visualizan la importancia de la

Cuadro 2

Importancia de la gestión del conocimiento

\begin{tabular}{ll}
\hline \multicolumn{1}{c}{ Autor } & \multicolumn{1}{c}{ Importancia } \\
\hline Koulopoulos y Frappaolo (2000) & $\begin{array}{l}\text { Herramienta fundamental para apoyar el crecimiento de } \\
\text { las organizaciones, tomando como punto de partida la sa- } \\
\text { biduría colectiva }\end{array}$ \\
Martín Rubio y De La Calle (2003) & $\begin{array}{l}\text { Valor generado y no acumulación de conocimiento } \\
\text { Creación de conocimiento para transmitirlo a toda la or- } \\
\text { ganización }\end{array}$ \\
\hline
\end{tabular}

Fuente: Elaboración propia. 
Intermediación de conocimiento: proceso de la gestión del conocimiento ...

Flores Urbáez, Matilde y Ramírez Omaña Rosa

gestión del conocimiento como un proceso organizacional dirigido a crear una cultura del compartir, generar, orientar, aplicar y evaluar el conocimiento; esto con la finalidad de ser aplicado por todos los miembros de la organización para tomar decisiones asertivas en entornos complejos, incrementar la productividad de la organización, así como generar procesos, productos y servicios innovadores.

Este enfoque está en consonancia con la razón de ser de las empresas cooperativas y deja ver la necesidad de generar conocimiento en equipo, punto clave para el crecimiento de las organizaciones de esta naturaleza.

\section{Modelo de gestión del conocimiento de Koulopoulos y Frappaolo (2000)}

Los procesos de la gestión del conocimiento de este modelo son: intermediación del conocimiento, exteriorización del conocimiento, interiorización del conocimiento y cognición. Este trabajo forma parte de uno más extenso, en el cual se evaluaron todos los subprocesos de la gestión del conocimiento del modelo teórico de Koulopoulos y Frappaolo, por lo tanto, para poder mostrar la importancia de cada uno en detalle, publicaremos los subprocesos por separado iniciando con la intermediación del conocimiento. En este estudio se abordará el proceso de intermediación de conocimiento.

La intermediación como proceso de creación de conocimiento, citando a Sanguino (2003), queda claramente establecida en la premisa de que las organizaciones son responsables de crear conocimiento, facilitar las condiciones que permitan un ambiente favorable para actividades en grupo y para generar y acumular conocimiento a nivel individual.

La intermediación de conocimiento debe promover la conexión entre las personas para que puedan acceder al conocimiento, generarlo y además transformarlo en innovaciones. Para ello, es importante promover la creación de equipos de trabajo y el compromiso con el cambio. Estos equipos deben actuar en un contexto multidisciplinario, en un clima organizacional que permita incentivar las capacidades de innovación y facilite el desarrollo de competencias a partir de la actividad misma de trabajar en equipo.

La gestión del conocimiento como macroproceso debe asumir la función de proveer a la organización de una plataforma que permita conectar a las personas con la fuente del conocimiento a través del fomento de relaciones de colaboración entre las personas involucradas y, entre otras cosas, la promoción de encuentros que permitan compartir el conocimiento tácito ${ }^{1}$ de acuerdo a las necesi-

1 Se entiende por conocimiento tácito aquel que es muy personal y difícil de plantear a través del lenguaje formal, por lo que resulta difícil transmitirlo y compartirlo con otros. La intuición, las ideas y las corazonadas subjetivas son parte de él. Este tipo de conocimiento tiene sus raíces en lo más profundo de las acciones y la experiencia individual, así como en los ideales, valores y emociones de cada persona (Nonaka y Takeuchi, 1995:7). 
dades de cada uno. Esto amerita conocer el perfil de cada individuo para impulsar los mejores encuentros en términos de la difusión del conocimiento.

En otras palabras, tal y como lo señalan Koulopoulos y Frappaolo (2000), la organización debe propiciar un encuentro entre el conocimiento y las personas y conectar al buscador de conocimiento con la fuente óptima para ese buscador.

En este contexto los autores plantean que la intermediación de conocimiento puede ser de dos tipos: asincrónica y sincrónica.

\section{A. Intermediación asincrónica}

Se caracteriza porque la exteriorización y la interiorización ${ }^{2}$ no ocurren en forma simultánea (asincronía). Aquí cobran importancia los bancos externos de datos, en los que se clasifica y almacena conocimiento e información acerca de los propietarios del conocimiento y corresponden a la fuente de la cual los individuos extraen conocimiento explícito relevante aún antes de que surja una necesidad o problema a resolver dentro de la organización.

Resaltan Koulopoulos y Frappaolo que básicamente la intermediación asincrónica se basa en identificar los posee- dores de conocimiento tácito, quienes difícilmente acceden a plasmarlo en documentos que puedan ser almacenados y recuperados en el momento adecuado. El conocimiento explícito es más fácil de transmitir y se puede conseguir con facilidad, pero a menudo el conocimiento tácito es más valioso para una organización, por lo tanto, ésta debe impulsar su transferencia como herramienta poderosa para mejorar la productividad de la organización.

\section{B. Intermediación sincrónica}

Este tipo de intermediación se presenta cuando la exteriorización y la interiorización ocurren en forma simultánea. La función primordial de la organización se centra en conectar al proveedor con el buscador de conocimiento. En este caso el conocimiento no se almacena sino que se transfiere mediante comunicación directa, lo cual es mucho más frecuente que ocurra en las agrupaciones informales de individuos, unidos por un interés o vocación, como en las comunidades de práctica, consideradas como el mecanismo más efectivo para la generación e intercambio de conocimiento dentro de la organización.

2 La interiorización del conocimiento, como la define Ruiz (2002), es el fruto del aprendizaje y lo vincula directamente con las políticas de formación que se aplican en las organizaciones, orientadas a la preparación relacionada con el puesto que desempeña y otras de formación humana y técnica mas generales, en general, todas las actividades que generan aprendizaje continuamente se convierten en experiencias que ayuda a interiorizar conocimiento sean o no promovidas por la empresa. La exteriorización desde el punto de vista de Muñoz y Riverola (2003), se constituye en la materialización del conocimiento y se entiende como su transformación en una forma que pueda ser manipulada, almacenada, transmitida, recuperada y utilizada fácilmente sin tener que recurrir a la persona que lo originó. Una etapa previa a esta actividad está signada obligatoriamente por un inventario de conocimientos de la empresa que consiste en la observación de problemas y disponibilidad de conocimiento. 
Intermediación de conocimiento: proceso de la gestión del conocimiento ...

Flores Urbáez, Matilde y Ramírez Omaña Rosa

\section{Intermediación de conocimiento en cooperativas agrarias del municipio La Cañada de Urdaneta.}

En el municipio La Cañada de Urdaneta operan nueve cooperativas agrarias, de su estudio los resultados en torno a la intermediación de conocimiento son los que a continuación se presentan:

En relación a la cantidad de poseedores de conocimiento tácito, los resultados revelan que los 15 entrevistados lograron identificar 66 personas poseedo- ras de conocimiento tácito en sus respectivas organizaciones. Tal y como se observa en la Tabla 1, en las cooperativas estudiadas, hay un predominio de poseedores de conocimiento en el área agropecuaria, lo cual es lógico dada su naturaleza. Sin embargo, aunque en menor cantidad se identificaron áreas no agropecuarias, es interesante la incorporación de personas con otros conocimientos no asociados directamente con la actividad económica de la organización pero que contribuyen a su funcionamiento.

Tabla 1

Cantidad de poseedores de conocimiento tácito

\begin{tabular}{|c|c|c|}
\hline Categoría de análisis & Categoría de respuesta & Frecuencia \\
\hline \multirow{19}{*}{$\begin{array}{l}\text { Identificación de los } \\
\text { poseedores del } \\
\text { conocimiento tácito }\end{array}$} & Agricultor & 66 \\
\hline & Salud animal & 2 \\
\hline & Pastos & 1 \\
\hline & Manejo de ganadería vacuna & 1 \\
\hline & Manejo de ganadería ovina & 1 \\
\hline & Manejo de ganadería porcina & 1 \\
\hline & Manejo ganadería caprina & 1 \\
\hline & Enfermería & 1 \\
\hline & Derecho & 1 \\
\hline & Mecánico & 1 \\
\hline & Albañil & 4 \\
\hline & Compra - venta de alimentos & 1 \\
\hline & Transporte de carga pesada & 1 \\
\hline & Mercadeo & 1 \\
\hline & Lombricultura & 1 \\
\hline & Ingeniero civil & 1 \\
\hline & Ventas & 1 \\
\hline & Cocina & 1 \\
\hline & TOTAL & 66 \\
\hline
\end{tabular}

Fuente: Elaboración propia. 
Aún cuando en la tabla no se expresa si el conocimiento de esas personas está clasificado en bases de datos, más adelante se verá que en algunos sí lo están, pero en bases de datos manuales. Los resultados obtenidos evidencian que en las cooperativas estudiadas está presente uno de los indicadores de la intermediación asincrónica: la identificación de los poseedores de conocimiento tácito.

Respecto al banco de almacenamiento de datos, Koulopoulos y Frappaolo (2000) definen un banco externo de conocimiento como aquel que almacena información acerca de los propietarios de conocimiento. Cuando un buscador de conocimiento necesita de él, la base de conocimiento puede buscarse y el conocimiento relevante se puede extraer.

Sin embargo en las cooperativas estudiadas, tal y como se observa en la Tabla 2, las bases de conocimiento identificadas por los entrevistados fueron predominantemente los libros de actas. Este método de almacenamiento no es adecuado debido a que los libros de actas son de acceso restringido. Otra limitante encontrada, está en que una parte importante de los entrevistados respondieron que el conocimiento se almacena en la mente de las personas, lo que obviamente presenta problemas, pues es difícil obtener y depende de la voluntad del poseedor para transferirlo a quien lo solicite.

A pesar de lo anterior, el hecho de que haya cooperativas que utilicen guías de trabajo, libros de diario y cuadernos de notas para almacenar conocimiento, representa un aspecto positivo, debido a que los mismos son más accesibles para los asociados. Solo en este caso los resultados coinciden con la propuesta de Koulopoulos y Frappaolo en cuanto a la presencia de intermediación asincrónica.

Asimismo, Koulopoulos y Frappaolo (2000) señalan que en una organización es mucho más común la transferencia de conocimiento explícito a través de bases de datos que la transferencia de conocimiento tácito mediante la comunicación directa, la cual permite un diálogo mas complejo y enriquecedor beneficiando a cada una de las partes involucradas.

En todas las cooperativas estudiadas, tal y como se observa en la Tabla 3, se da la transferencia de conocimiento tácito a través de la comunicación directa en reuniones de trabajo. La acción de

Tabla 2

Banco de almacenamiento de conocimiento

\begin{tabular}{llc}
\hline Categoría de análisis & Categoría de respuesta & Frecuencia \\
\hline Bancos de almacenamiento & Guías de trabajo & 5 \\
de conocimiento & Los libros de actas & 12 \\
& Libro de diario & 1 \\
& Cuaderno de notas & 1 \\
& En la mente de cada de cada uno & 6 \\
& TOTAL & 25 \\
\hline
\end{tabular}

Fuente: Elaboración propia. 
Intermediación de conocimiento: proceso de la gestión del conocimiento ...

Flores Urbáez, Matilde y Ramírez Omaña Rosa

Tabla 3

Transferencia del conocimiento mediante comunicación directa

\begin{tabular}{llc}
\hline Categoría de análisis & Categoría de respuesta & Frecuencia \\
\hline $\begin{array}{l}\text { Transferencia del conocimiento } \\
\text { mediante comunicación directa }\end{array}$ & Reuniones de trabajo & 15 \\
\hline Fuente: Elaboración propia. & &
\end{tabular}

propiciar encuentros entre las personas involucradas en la organización, amerita conocer el perfil de cada uno de ellos. Para las cooperativas esto es posible debido a que según lo mostrado la tabla, resultó que las personas además de conocer el oficio que cada uno de los asociados, ellos mismos impulsan encuentros y reuniones en términos para discutir ideas y opiniones sobre un tema, lo cual se genera en un proceso de intercambio de conocimiento.

Sin embargo, la difusión del conocimiento en forma de comunicación directa, no debe quedarse solo en reuniones de trabajo, es necesario como lo expone Gunnlaugsdottir (2003, citado por García y Cordero, 2008), promover la creación de equipos de trabajo comprometidos con el cambio, directamente encaminados a mejorar su desempeño final guiado por objetivos claros.

Por otra parte, la propuesta teórica de Koulopoulos y Frappaolo (2000) identifica a la agrupación informal de individuos unidos por un interés común, como una vía para reforzar la intermediación sincrónica del conocimiento entre socios movidos por un interés común.

En tal sentido, en el presente estudio, tal como se muestra en la Tabla 4, los entrevistados mostraron una marcada tendencia a promocionar estos encuentros en función de intercambiar conoci- miento, seguido de "mejorar la administración". Esto revela indicios de una conciencia colectiva de compartir, típica de la gerencia del conocimiento, tal y como lo plantean Koulopoulos y Frappaolo (2000), quienes resaltan que una de las funciones de la gerencia del conocimiento se centra en el apalancamiento de la sabiduría colectiva para aumentar la capacidad de respuesta y la innovación.

Con respecto a la conexión del buscador de conocimiento con el proveedor de conocimiento, forma parte de las herramientas importantes para lograr la intermediación sincrónica de conocimiento (Koulopoulos y Frappaolo, 2000). Más aún cuando en las cooperativas en estudio, los entrevistados lograron identificar en qué se especializaban sus compañeros de trabajo, tal como se mostró en la Tabla 1. Esto facilita notablemente el trabajo de vincular a la gente, para lograr la transferencia de conocimiento en forma directa y sincrónica.

Ahora bien, como se evidencia en la Tabla 5, la mayor frecuencia de respuestas se obtuvo en la opción buscar conocimiento dentro de la cooperativa, lo que resalta con fuerza la afirmaciones de Koulopoulos y Frappaolo (2000), en cuanto a que las organizaciones encaminan sus esfuerzos a obtener conocimiento de sus sistemas internos de gerencia y muy pocos a introducir conocimientos al sistema proveniente del entorno. 
Tabla 4

Agrupación informal de individuos unidos por un interés común

\begin{tabular}{llc}
\hline Categoría de análisis & Categoría de respuesta & Frecuencia \\
\hline & Para intercambiar conocimiento & 16 \\
& Para mejorar la administración & 4 \\
Agrupación informal & Resolver problemas & 3 \\
de individuos unidos & Nombrar junta directiva & 1 \\
por un interés común & Remover o sancionar socios & 1 \\
& Buscar financiamiento & 1 \\
& Compartir con los compañeros & 2 \\
& Mejorar las relaciones & 1 \\
\hline
\end{tabular}

Fuente: Elaboración propia.

Tabla 5

Conexión del buscador de conocimiento con el proveedor de conocimiento

\begin{tabular}{llc}
\hline Categoría de análisis & Categoría de Respuesta & Frecuencia \\
\hline Conexión del buscador del & Buscan proveedores de conocimiento & 16 \\
conocimiento con el proveedor & dentro de la cooperativa & \\
del conocimiento & Buscan fuera de la cooperativa. & 10 \\
& Asisten a cursos, charlas y conferencias & \\
\hline
\end{tabular}

Fuente: Elaboración propia.

\section{Conclusiones}

Aún cuando los resultados obtenidos no pueden generalizarse como un principio universal, sí pueden servir de punto de partida para introducir cambios que permitan de manera progresiva construir un proceso de intermediación de conocimiento adecuado a la naturaleza de las organizaciones estudiadas y promover la economía social y participativa representada por este tipo de organizaciones.

Dada la importancia de almacenar el conocimiento en bases de datos de fácil acceso a todos los asociados de la cooperativa, es recomendable sustituir los libros de actas por otro mecanismo de almacenamiento más accesible, igual se aplica al libro del diario y a los cuadernos de notas.

Ahora bien, las cooperativas estudiadas en su afán de proveerse de conocimiento, miran a su interior, dando cabida a la obsolescencia de los asociados. Es necesario el reabastecimiento permanente de conocimiento, logrado a través de políticas de formación técnica, encaminadas a mirar su entorno y lograr el máximo aprovechamiento de oportunidades que ofrece el mercado.

A pesar de que buscan auto abastecerse en materia de conocimiento, es 
Intermediación de conocimiento: proceso de la gestión del conocimiento ...

Flores Urbáez, Matilde y Ramírez Omaña Rosa

contradictorio que la mayoría de las cooperativas no lo almacenen en forma física, lo relativo al conocimiento que poseen, ni la formación que han tenido o la experiencia acumulada. Esta práctica debe implementarse como norma, para encaminar esfuerzos hacia la obtención del conocimiento que cada uno posee, para beneficio colectivo, además de guiar a la organización en el conocimiento de las necesidades de capacitación de cada uno en particular.

Una de las herramientas imprescindibles en la formación de los asociados es la literatura especializada o material de apoyo didáctico, por cuanto esta práctica se traduce en enriquecimiento del conocimiento personal. En base a esto la organización puede promocionar esta actividad como medio de crecimiento, aprovechando las reuniones de trabajo para discutir temas que no hayan sido totalmente comprendidos por algún lector o para transmitir el conocimiento adquirido a todos sus compañeros.

En consecuencia, si las cooperativas no actualizan sus stocks de conocimiento, no utilizan material didáctico de apoyo, les resultará muy difícil organizar sus ya incipientes bases de datos, lo que implica que no puedan acceder a ellas con la ligereza que el caso amerite y por tanto el conocimiento acorde con el proceso, según los informantes clave, no será suficiente para la toma de decisiones.

Finalmente, es importante internalizar el concepto de gestión del conocimiento como un enfoque de gestión organizacional que involucra y compromete a todos sus individuos, implica trabajar en equipo, compartir conocimientos, asumir compromisos que generen una nueva cultura en la organización, que facilite compartir todos los tipos de conocimientos que conlleven a realizar las metas y objetivos de la organización de la forma más eficaz posible.

\section{Referencias bibliográficas}

Alianza Cooperativa Internacional -ACl(1995). Declaración de la Alianza. Cooperativa Internacional sobre la Identidad Cooperativa. Manchester - Reino Unido, disponible en http://www.elhogarobrero1905.org.ar/, consultado el 14 de mayo de 2010.

Asamblea Nacional de la República Bolivariana de Venezuela (1999). Constitución de la República Bolivariana de Venezuela.

Bustamante Salazar, Alina Marcela (2009). "Cooperativismo de trabajo asociado y estrategia. Revisión de literatura", Revista Pensamiento \& Gestión, 27, Universidad del Norte, 201-234, Colombia.

Comité Ejecutivo de la Organización Internacional de Cooperativas de Producción Industrial, Artesanal y de Servicios -CICOPA- (2005). Declaración Mundial sobre Cooperativismo de Trabajo Asociado, Oslo, disponible en http://www.cicopa.coop/, consultado el 10 de junio de 2011.

Fransi, Eduard Cristóbal; Montegut Salla, Yolanda y Marimon i Viadiu, Frederic (2007). "La gestión de las cooperativas agrarias: tipificación de las cooperativas del sector oleícola de Catalunya", CIRIEC - España, Revista de Economía Pública, Social y Cooperativa, disponible en http://search. ebscohost.com/login.aspx? Direct=tr $\mathrm{ue} \& \mathrm{db}=\mathrm{zbh} \& \mathrm{AN}=$ 31816792\&lang= es\&site=ehost-live, consultado el 10 de junio de 2011. 
García, Francisco y Cordero, Ana Emilia (2008). La gestión del conocimiento y los equipos de trabajo. Observatorio Laboral, Revista Laboral, volumen 1 , número 2, julio-diciembre, Universidad de Carabobo, Venezuela.

Hernández, Roberto; Fernández, Carlos y Baptista, Pilar (2006). Metodología de la Investigación, Editorial Mc Graw - Hill, México.

Koulopoulos, Thomas y Frappaolo, Carl (2000). Lo fundamental y lo más efectivo acerca de la gerencia del conocimiento, Editorial McGraw-Hill Interamericana, S.A. Bogotá, Colombia.

Ley Especial de Asociaciones Cooperativas (2001). Disponible en www.bvsst.org. Ve/documento/pnf/ley_especial_de_ asociaciones_cooperativas.pdf, consultado el 20 de abril de 2010.

Martínez, Miguel (2001). El comportamiento Humano, Trillas, México.

Martín Rubio, Irene y De La Calle, Carmen (2003). Evolución de la gestión del conocimiento hacia la creación de valor. Estudio de un caso, Revista Madr+d. Nro. 17, junio. Disponible en http://www.madrimasd.org/revista/ revista17/aula/aula2.asp. Consultado el 4 de febrero de 2008.

Méndez, Carlos (2001). Metodología. Diseño y Desarrollo del Proceso de Investigación. Editorial McGraw-Hill Interamericana, S.A. Bogotá, Colombia.

Ministerio de Ciencia y Tecnología (2005). Plan Nacional de Ciencia, Tecnología e Innovación 2005-2030.

Ministerio del Poder Popular para la Comunicación y la Información, MINCl, Cadivi aprobó $\$ 5.843$ millones para la importación de alimentos, 4-12008, disponible en http://www.minci.gob.ve/pagina/1/17264/cadivi_ aprob5.843_millones.html, consultado el 20 de marzo de 2008.
Ministerio del Poder Popular para la Comunicación y la Información, MINCI Foncrei ha otorgado Bs.F. 257 millones en créditos a cooperativas desde 2005, Noticias Economía, 17-03-2008, disponible en http://www.minci.gob.ve/a_r_r/1/175 664/foncrei_ha_otorgado.prnt, consultado el 20 marzo 2008).

Muñoz Seca, Beatriz y Riverola, Josep (2003). Del buen pensar y mejor hacer, Editorial McGraw-Hill, Interamericana de España, S.A.U., Madrid, España.

Nonaka, Ikujiro y Takeuchi, Hirotaka (1995). La organización creadora de conocimiento. Cómo las compañías japonesas crean la dinámica de la innovación. Oxford University Press. México.

Presidencia de la República Bolivariana de Venezuela. Proyecto Nacional Simón Bolívar. Desarrollo Económico y Social de la Nación 2007-2013.

Ruiz Llavero, Gustavo (2001). Orígenes y utilización actual de la gestión del conocimiento, DTI Consultores, http://www. dti.es/artículos/PDF/KM2.pdf. Consultado el 18/12/2009.

Salazar, Alina y Bustamante, Marcela (2009). "Cooperativismo de trabajo asociado y estrategia: revisión de literatura". Revista: Pensamiento \& Gestión; pp. 201-234, 34p. Colombia.

Sanguino, Ramón (2003). La Gestión del conocimiento. Su importancia como recurso estratégico para la organización, disponible en 5campus.org, Marketing http://www.5campus.org/ leccion $/ \mathrm{km}$ [Consultado el 08/10/ 2008].

SUPERINTENDENCIA DE COOPERATIVAS DE VENEZUELA (2001). Ley Especial de Asociaciones Cooperativas, Venezuela, consultado el 20 de marzo de 2010, disponible en http://www.sunacoop.gob.ve 